\title{
PLATAFORMA COMPUTACIONAL PARA ANÁLISE DA TRANSFERÊNCIA DE CALOR BIDIMENSIONAL
}

COMPUTATIONAL PLATFORM FOR ANALYSIS OF TWO-DIMENSIONAL HEAT TRANSFER

Marília Pereira Silva ${ }^{1}$, Gislane Pinho de Oliveira ${ }^{2}$, Raimundo Nonato Diniz Costa Filho ${ }^{3}$

DOI: 10.37702/REE2236-0158.v39p390-399.2020

\begin{abstract}
RESUMO
A transferência de calor visa a estudar o fluxo de calor e é uma disciplina que integra a grande curricular de vários cursos de Engenharia. Existe uma ampla disponibilidade de livros-texto nesta área; porém, há poucos softwares open source destinados ao ensino desta disciplina. Neste contexto, o presente trabalho apresenta uma ferramenta computacional do tipo open source desenvolvida em ambiente MATLab. O principal objetivo do software é facilitar o processo de aprendizagem do aluno, fazendo uma ponte entre a teoria e o mundo prático. O software permite a exibição de gráficos $2 \mathrm{D}$ e $3 \mathrm{D}$, para uma geometria retangular em regime permanente e transiente, em função das características do material e das temperaturas em contato com o objeto. Nesta ferramenta considerou-se que a placa estaria em contato com um fluido, necessariamente na sua superfície lateral superior, o que deve ser analisado com cautela no momento de inserção dos dados iniciais.
\end{abstract}

Palavras-chave: transferência de calor; simulação computacional; software.

\begin{abstract}
Heat transfer aims to study heat flow and is a subject that integrates the syllabus of various engineering courses. There is a wide availability of textbooks in this area, but there are few open source software for teaching this subject. In this context, the present work presents an open source computer tool developed in MATLab environment. The main purpose of the software is to facilitate the student learning process by bridging theory and the practical world. The software allows the display of 2D and 3D graphics for rectangular geometry in steady-state and transient regime, depending on the material characteristics and the temperatures in contact with the object. In this tool it was considered that the plate would be in contact with a fluid, necessarily on its upper lateral surface, which should be carefully analyzed at the time of initial data insertion.
\end{abstract}

Keywords: heat transfer; computer simulation; software.

\section{INTRODUÇÃO}

A transferência de calor está estritamente relacionada aos processos energéticos e à conservação e produção de energia. A crescente integração de novas tecnologias torna indispensável explorar os processos térmicos (INCROPERA; DEWITT; BERGMAN, 2008).
Compreender tais processos é determinante para o desempenho do profissional de engenharia, visto que muitos equipamentos são projetados principalmente com base na análise da transferência de calor, como trocadores de calor, caldeiras, condensadores, radiadores, aquecedores, fornos, refrigeradores, coletores de energia,

\footnotetext{
${ }^{1}$ Graduada, Universidade Federal do Maranhão, Campus Balsas, marilia28_ps@ hotmail.com

${ }^{2}$ Profa. Me., Universidade Federal do Maranhão, Campus Balsas, gislane.oliveira@ufma.br

${ }^{3}$ Prof. Dr., Universidade Federal do Maranhão, Campus Balsas, raimundo.diniz@ufma.br
} 
além de outras várias aplicações na área industrial (ÇENGEL; GHAJAR, 2012).

A complexidade dos problemas dessa área exige o uso de mecanismos computacionais. Os métodos numéricos surgem como forma de otimização, objetivando rapidez e baixo custo, de problemas trabalhosos de serem resolvidos analiticamente. Os métodos numéricos mais utilizados para problemas de transferência de calor são o das diferenças finitas e o dos elementos finitos. Esses métodos são fundamentados na metodologia de malha discretizada, limitando-se à geometria do problema (ZIN CHO, 2011).

Todavia, tratando-se de problemas com geometrias complexas, é essencial o uso de métodos numéricos para minimizar o preenchimento de vetores e matrizes. Além disso, a dificuldade da manipulação de equações diferenciais, principalmente para o caso bidimensional, intensifica o uso dos métodos numéricos, como recurso para geração de soluções impossíveis de serem resolvidas analiticamente.

Assim, o propósito do uso de softwares, como ferramentas didáticas para o ensino de Engenharia, é facilitar o processo de aprendizagem e assim possibilitar o aperfeiçoamento do conteúdo adquirido durante aulas teóricas. Ademais, tal uso permite o desenvolvimento de novas formas de pensar, tornando-se relevante para que os alunos absorvam o conteúdo abordado, de forma clara e objetiva, o que, por sua vez, torna a aprendizagem dinâmica e isso contribui de maneira significativa para agregar valor à formação dos discentes.

Nesse contexto, há uma carência em softwares destinados ao estudo da transferência de calor. De acordo com Silva, Silva e Lima (2006), os poucos pacotes disponíveis no mercado - como por exemplo o conhecido ANSYS-CFX® (ANSYS CFX, 2019) - são muito caros e relativamente complicados, impossibilitando o seu uso por parte de alunos que estão iniciando no tema. Ademais, esses programas comerciais não possuem o código aberto, consequentemente, não é possível personalizar o programa, verificar a qualidade do código fonte, realizar melhorias no programa e corrigir erros. Nesse cenário, a ideia de software livre e de código aberto (FOSS Free and Open Source Software) é uma boa alternativa para os problemas apresentados nos programas de caráter comercial.

$\mathrm{Na}$ literatura especializada alguns FOSS desenvolvidos para a simulação de problema de transferência de calor foram encontrados. O TransCal foi desenvolvido pela equipe do professor Maliska para auxiliar no ensino de disciplinas relacionadas com transferência de calor e mecânica dos fluidos. O TransCal foi implementado em linguagem de programação C++ (MALISKA, 1998).

O CFD Sinflow é um software que permite a simulação numérica de problema 2D envolvendo escoamentos e transferência de calor. Esse software foi implementado em C++ (PIERITZ et al., 2004).

No trabalho de Silva, Silva e Lima (2006) é apresentado o software 1D HeatTransfer para simulações de problemas difusivos-conectivos de transferência de calor, porém em ambientes unidimensionais. $\mathrm{O}$ 1D HeatTransfer foi implementado em Fortran 95.

Diferente dos FOSS para transferência de calor citados anteriormente, este artigo apresenta um software desenvolvido em ambiente MATLab (MATrix Laboratory). Esse ambiente de programação foi escolhido por possuir várias vantagens quando comparado com outras linguagem de programação, por exemplo a facilidade de utilização, pois é uma linguagem interpretada; é uma linguagem bem eficiente para complexos cálculos matemáticos e manipulações numéricas de matrizes; possui um vasto número de funções, manipulações e alterações com estruturas; e, além de ser capaz de gerar gráficos com alta qualidade, o $M A T L A b$ possui um ambiente de construção de interface gráfica através do usuário (GUI) (LIMA; SILVA; LIMA, 2006). O software desenvolvido permite a simulação de problemas de transferência de calor, em regime permanente e transiente, a fim de se obter a distribuição de temperatura em uma placa retangular, encontrando-se a superfície superior em contato com um fluido.

O presente trabalho apresenta um diferencial no que diz respeito ao desenvolvimento das equações de diferenças finitas acopladas ao Método do Balanço da 
Energia, para uma barra metálica de dimensões diferentes nas direções $x$ e $y$. Outra característica típica corresponde ao desenvolvimento de um software para aprimorar a perspectiva quanto à distribuição de temperatura de determinado material, através das equações deduzidas.

Tendo em vista que os softwares mais utilizados para análise da transferência de calor são pagos e de difícil manipulação, desenvolveu-se este código-fonte, livre e passível de aprimoramentos, com o intuito de contribuir ao acervo das ferramentas didáticas educativas ligadas a esta área, assim como, estimular os estudantes de modelagem computacional.

\section{METODOLOGIA}

\section{Método das Diferenças Finitas}

Definindo-se as taxas de transferência de calor para um volume de controle, a partir da Lei de Fourier, e admitindo-se condutibilidade térmica constante em todas as direções, caracterizando o meio como isotrópico, tem-se a forma geral da equação do calor, dada pela Equação (1) (INCROPERA; DEWITT; BERGMAN, 2008).

$$
\frac{\partial^{2} T}{\partial x^{2}}+\frac{\partial^{2} T}{\partial y^{2}}+\frac{\partial^{2} T}{\partial z^{2}}+\frac{\dot{q}}{k}=\frac{1}{\alpha} \frac{\partial T}{\partial t}
$$

em que $\alpha=\frac{k}{\rho C_{p}}$ é a difusibilidade térmica, que indica a relação entre a capacidade do material em transferir calor e a capacidade de armazenar energia térmica (INCROPERA; DEWITT; BERGMAN, 2008).

Em regime permanente o lado direito da Equação (1) será nulo. Ademais, o problema tratado neste trabalho não possui geração interna e varia somente nas direções $x$ e $y$. Sendo assim, utiliza-se o Método das Diferenças Finitas para determinar as derivadas parciais de segunda ordem com relação à $x$ e à $y$ exibidas no lado esquerdo da equação.

Para análise numérica, inicialmente, faz-se a distinção dos pontos nodais, com a subdivisão do meio de interesse, em um número de pequenas regiões, estabelecendo para cada uma um ponto de referência fixado no seu centro, denominado nó ou ponto nodal. Os pontos nodais são nomeados por um esquema de numeração, na qual as posições $x$ e $y$ são índices $m$ e $n$, respectivamente (INCROPERA; DEWITT; BERGMAN, 2008). Ademais, para a determinação numérica da distribuição da temperatura, é necessária uma equação de conservação conveniente para cada um dos pontos nodais de temperaturas não conhecidas, fazendo-se o uso da aproximação por diferenças finitas (INCROPERA; DEWITT; BERGMAN, 2008).

Considerando-se que a aproximação para a derivada segunda, no ponto nodal $m, n$ é dada por:

$$
\left.\frac{\partial^{2} T}{\partial x^{2}}\right|_{m, n} \approx \frac{\partial T /\left.\partial x\right|_{m+1 / 2, n}-\partial T /\left.\partial x\right|_{m-1 / 2, n}}{\Delta x}
$$

as taxas de variação de temperatura podem ser definidas em razão das temperaturas nodais, isto é:

$$
\begin{aligned}
& \left.\frac{\partial T}{\partial x}\right|_{m+1 / 2, n} \approx \frac{T_{m+1, n}-T_{m, n}}{\Delta x} \\
& \left.\frac{\partial T}{\partial x}\right|_{m-1 / 2, n} \approx \frac{T_{m, n}-T_{m-1, n}}{\Delta x}
\end{aligned}
$$

Assim, substituindo as Equações (3) e (4) na Equação (2), tem-se a aproximação por diferenças finitas na direção $x$.

$$
\left.\frac{\partial^{2} T}{\partial x^{2}}\right|_{m, n} \approx \frac{T_{m+1, n}+T_{m-1, n}-2 T_{m, n}}{(\Delta x)^{2}}
$$

De forma semelhante, obtém-se a Equação (6) para a direção $y$.

$$
\left.\frac{\partial^{2} T}{\partial y^{2}}\right|_{m, n} \approx \frac{T_{m, n+1}+T_{m, n-1}-2 T_{m, n}}{(\Delta y)^{2}}
$$

\section{Método do Balanço de Energia}

Neste método, aplica-se a conservação de energia em uma superfície de controle para a obtenção da equação de diferenças finitas de um ponto nodal, considerando-se que todos os fluxos térmicos são orientados para o interior 
do ponto, visto que a orientação real do fluxo é desconhecida.

Para um plano bidimensional, em regime estacionário, sem geração de energia, determinando-se uma superfície de controle em torno do ponto nodal interior $m, n$, a transferência de calor ocorre por meio da condução entre $m, n$ e os seus quatro nós adjacentes, logo:

$$
\sum_{i=1}^{4} q_{(i) \rightarrow(m, n)}=0
$$

Observando-se a Figura 1 e supondo-se que a energia transferida por condução se dá por toda a extensão das faixas orientadas nas direções $x$ e $y$, determina-se as expressões referentes às taxas de condução. Dessa maneira, a equação do nó $(m-1, n)$ para o nó $(m, n)$ é dada pela Equação (8):

$$
q_{(m-1, n) \rightarrow(m, n)}=k(\Delta y \cdot w)\left(\frac{T_{m-1, n}-T_{m, n}}{\Delta x}\right)
$$

em que o termo $(\Delta y \cdot w)$ é a área de transferência de calor e $\left(\frac{T_{m-1, n}-T_{m, n}}{\Delta x}\right)$ é a aproximação por diferenças finitas do gradiente de temperatura entre os dois pontos nodais.

Com isso, as equações referentes aos demais pontos nodais podem ser escritas da seguinte forma:

$$
\begin{aligned}
& q_{(m+1, n) \rightarrow(m, n)}=k(\Delta y \cdot w)\left(\frac{T_{m+1, n}-T_{m, n}}{\Delta x}\right) \\
& q_{(m, n+1) \rightarrow(m, n)}=k(\Delta y \cdot w)\left(\frac{T_{m, n+1}-T_{m, n}}{\Delta x}\right) \\
& q_{(m, n-1) \rightarrow(m, n)}=k(\Delta y \cdot w)\left(\frac{T_{m, n-1}-T_{m, n}}{\Delta x}\right)
\end{aligned}
$$

Figura 1 - Representação da condução para um ponto nodal interior a partir de seus pontos adjacentes

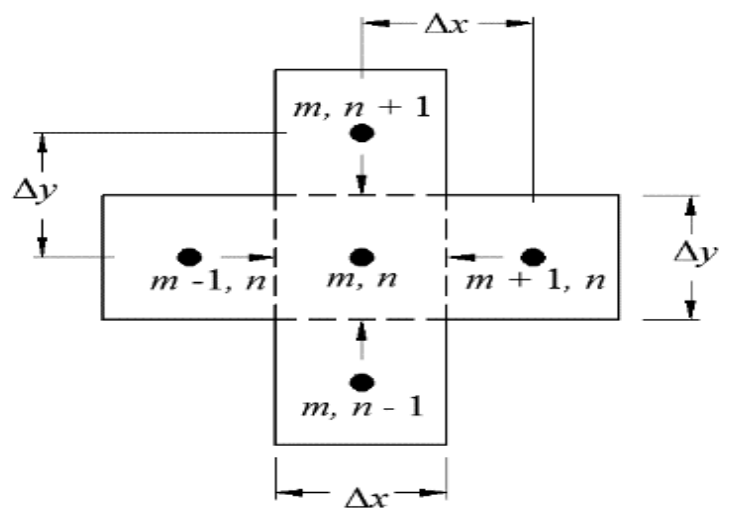

Fonte: Incropera; Dewitt; Bergman (2008).

Considerando-se $\Delta x \neq \Delta y$, obtém-se a equação de diferenças finitas para um nó interno:

$$
\begin{aligned}
T_{m-1, n}+T_{m+1, n}+ & \left(\frac{\Delta x^{2}}{\Delta y^{2}} T_{m, n-1}\right) \\
& +\left(\frac{\Delta x^{2}}{\Delta y^{2}} T_{m, n+1}\right) \\
& -2 T_{m, n}\left[1+\frac{\Delta x^{2}}{\Delta y^{2}}\right]=0
\end{aligned}
$$

Todavia, a Equação (12) requer que a temperatura do ponto nodal interior seja similar a uma média ponderada das temperaturas dos quatro pontos adjacentes.

É possível que a temperatura desconhecida esteja em uma superfície submetida à convecção; nesse caso, é necessária a formulação de novas equações para pontos submetidos a essa condição, exemplificada pela Figura 2.

Figura 2 - Representação do ponto nodal em superfície lateral superior com convecção

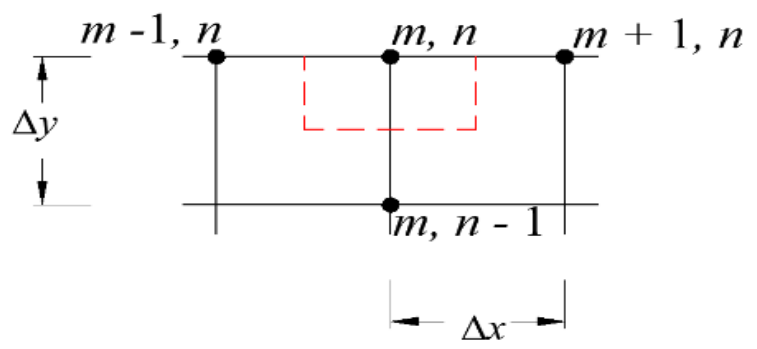

Fonte: Incropera; Dewitt; Bergman (2008). 
A transferência de calor por convecção é provocada pelo deslocamento da massa fluida, devido a um gradiente de temperatura, determinada pelo coeficiente $q_{c v y}$ :

$$
q_{c v}=h \cdot A \cdot\left(T_{\infty}-T_{s}\right)
$$

em que $h$ é o coeficiente de transferência de calor por convecção, A é a área, $T_{S}$ é a temperatura da superfície sólida e $T_{\infty}$ a temperatura do fluido.

Em regime permanente, sem geração de energia e $\Delta x \neq \Delta y$, obtém-se a equação de diferenças finitas para pontos nodais localizados em superfície lateral superior submetido a convecção:

$$
\begin{aligned}
T_{m-1, n}+T_{m+1, n}+2 & \left(\frac{\Delta x^{2}}{\Delta y^{2}} T_{m, n-1}\right) \\
& +\left(2 B i \cdot \frac{\Delta x}{\Delta y} T_{\infty}\right) \\
& +2 T_{m, n}\left[1-\frac{\Delta x^{2}}{\Delta y^{2}}-B i \frac{\Delta x}{\Delta y}\right] \\
& =0
\end{aligned}
$$

em que $B i$ é o número adimensional de Biot, que relaciona a transferência de calor por convecção com a condução, definido pela Equação (15):

$$
B i=\frac{h \Delta x}{k}
$$

Considerando-se uma placa retangular, sob condições de regime permanente, em plano bidimensional, condutibilidade térmica constante e ausência de geração de energia, na qual três superfícies possuem temperaturas constantes e uma superfície está em contato com um fluido adjacente a ela, conforme a Figura 3, nota-se que a malha é constituída por dois tipos de pontos nodais: pontos interiores e pontos em uma superfície plana com convecção. Logo, utilizando-se o Método do Balanço de Energia, baseado na conservação em um volume de controle, obtém-se as equações de diferenças finitas, possibilitando a determinação da distribuição de temperaturas.

O problema em questão pode ser representado pela notação matricial, conforme a Equação (16), em que é dado um sistema de $\mathrm{N}$ equações de diferenças finitas correspondente a $\mathrm{N}$ temperaturas desconhecidas. Desse modo, aplicou-se o Método da Inversão de Matriz para a sua resolução.

Figura 3 - Representação da malha utilizada no regime permanente

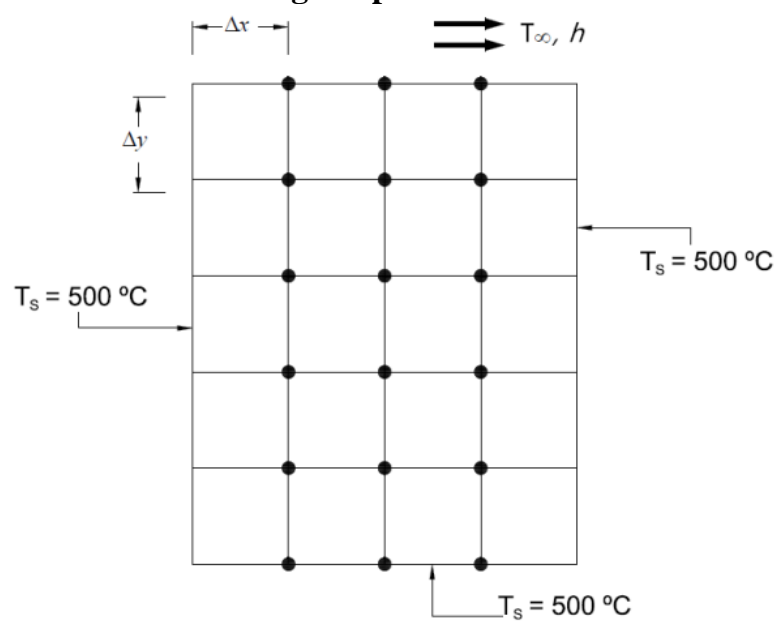

Fonte: elaborada pelos autores.

$$
\begin{gathered}
{[A][T]=[C]} \\
{\left[\begin{array}{ccc}
a_{11} & \cdots & a_{1 N} \\
a_{21} & \cdots & a_{2 N} \\
\vdots & \vdots & \vdots \\
a_{N 1} & \cdots & a_{N N}
\end{array}\right]\left[\begin{array}{c}
T_{1} \\
T_{2} \\
\vdots \\
T_{N}
\end{array}\right]=\left[\begin{array}{c}
C_{1} \\
C_{2} \\
\vdots \\
C_{N}
\end{array}\right]}
\end{gathered}
$$

em que $A$ é uma matriz quadrada $(N x N)$ na qual cada elemento dessa matriz dependerá do tipo de nó analisado e de seus adjacentes, e os vetores $T$ e $C$ são chamados de vetor solução e vetor do lado direito, respectivamente (INCROPERA; DEWITT; BERGMAN, 2008). É oportuno citar que cada linha da matriz $A$ possui no máximo cinco elementos (o nó de interesse e seus adjacentes) diferentes de zero e seus valores serão determinados em função do tipo de nó. No mínimo, cada linha terá um elemento não nulo, que será sempre pertencente à diagonal principal, referente ao nó de interesse, garantindo, dessa maneira, que a matriz não será, de forma alguma, singular, assegurando a aplicabilidade do Método da Inversão de Matriz para resolver qualquer sistema de equações lineares provenientes de problemas de calor.

\section{Discretização da Equação do Calor: Método Explícito}


Tomando-se a Equação (1) para um plano bidimensional, em regime transiente, sem geração de energia, tem-se a Equação (17):

$$
\frac{\partial^{2} T}{\partial x^{2}}+\frac{\partial^{2} T}{\partial y}=\frac{1}{\alpha} \frac{\partial T}{\partial t}
$$

Nos problemas transientes as temperaturas se alteram no decorrer do tempo, e a solução por diferenças finitas exige uma discretização no espaço e no tempo. Conforme Incropera, Dewitt e Bergman (2008), a aproximação por diferenças finitas para a derivada em relação ao tempo é definida pela Equação (18):

$$
\left.\frac{\partial T}{\partial t}\right|_{m, n} \approx \frac{T_{m, n}^{p+1}-T_{m, n}^{p}}{\Delta t}
$$

$\mathrm{O}$ inteiro $p$ expressa a dependência temporal da temperatura $T$ e a derivada em relação ao tempo é representada em termos da diferença entre as temperaturas relacionadas aos instantes de tempo novo $(p+1)$ e anterior $p$.

Se a Equação (18) for substituída na Equação (17), a característica da solução por diferenças finitas dependerá do instante de tempo em que as temperaturas estão sendo estabelecidas.

Definindo os termos do lado direito das Equações (5) e (6) em $p$ e substituindo na Equação (17), a forma explícita da equação de diferenças finitas para o nó interno $m, n$ é determinada pela equação a seguir:

$$
\begin{aligned}
& \frac{1}{\alpha} \frac{T_{m, n}^{p+1}-T_{m, n}^{p}}{\Delta t}=\frac{T_{m+1, n}^{p}+T_{m-1, n}^{p}-2 T_{m, n}^{p}}{(\Delta x)^{2}} \\
&+\frac{T_{m, n+1}^{p}+T_{m, n-1}^{p}-2 T_{m, n}^{p}}{(\Delta y)^{2}}
\end{aligned}
$$

Explicitando a temperatura nodal no novo instante de tempo $(p+1)$ e considerando $\Delta x \neq \Delta y$, obtém-se a Equação (20):

$$
\begin{aligned}
T_{m, n}^{p+1}=F o\left(T_{m+1, n}^{p}\right. & \left.+T_{m-1, n}^{p}\right) \\
& +F o\left(\frac{\Delta x^{2}}{\Delta y^{2}}\right)\left[\left(T_{m, n+1}^{p}\right.\right. \\
& \left.\left.+T_{m, n-1}^{p}\right)\right] \\
& +T_{m, n}^{p}[1-2 F o \\
& \left.-2 F o\left(\frac{\Delta x^{2}}{\Delta y^{2}}\right)\right]
\end{aligned}
$$

em que Fo é o número adimensional de Fourier, definido, em diferenças finitas, tal como a Equação (21):

$$
F o=\frac{\alpha \Delta t}{\left(\Delta x^{2}\right)}
$$

A equação definida anteriormente é explícita, pois as temperaturas nodais desconhecidas para o novo instante de tempo são estabelecidas unicamente por temperaturas nodais conhecidas no instante de tempo anterior (INCROPERA; DEWITT; BERGMAN, 2008).

Desse modo, o cálculo das temperaturas desconhecidas é direto, visto que a temperatura em cada ponto nodal é conhecida em $t=0$, os cálculos começam em $t=\Delta t(p=1)$. Com as temperaturas conhecidas em $t=\Delta t$, utiliza-se a equação de diferenças finitas correspondente para estabelecer a temperatura em $t=2 \Delta t$. Assim, a distribuição de temperatura transiente é obtida avançando no tempo, utilizando intervalos de $\Delta t$ (INCROPERA; DEWITT; BERGMAN, 2008).

A precisão da solução por diferenças finitas pode ser aperfeiçoada através da redução dos valores de $\Delta x$ e $\Delta t$. Evidentemente o número de pontos nodais interiores aumenta à medida que $\Delta x$ diminui, da mesma forma acontece para o número de intervalos de tempos necessários para gerar a solução, aumenta com a diminuição de $\Delta t$. Entretanto, o valor de $\Delta t$ é definido obedecendo a critérios de estabilidade e não de maneira aleatória (INCROPERA; DEWITT; BERGMAN, 2008). 
Figura 4 - Distribuição de temperatura no regime permanente

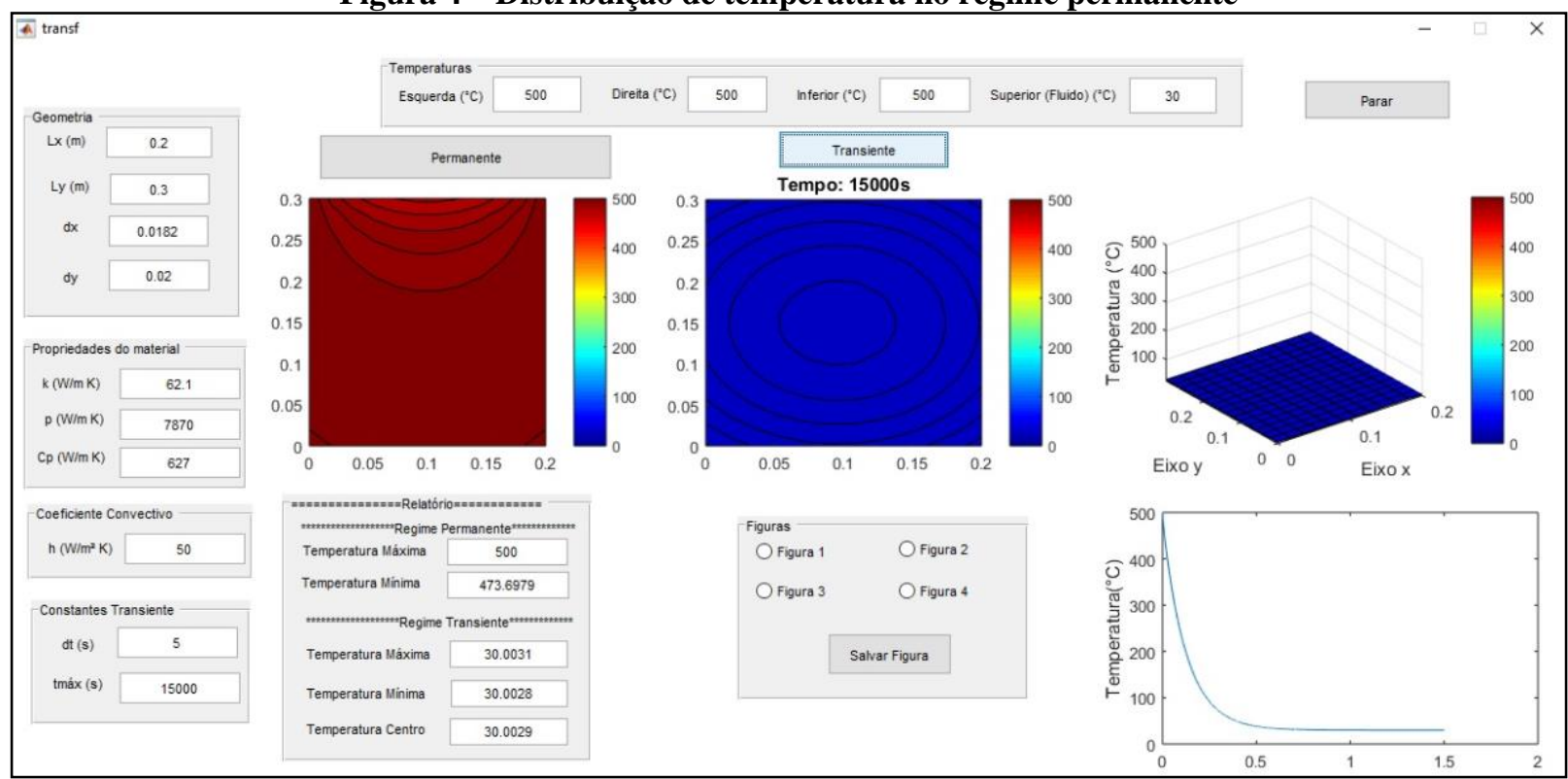

Fonte: acervo dos autores.

\section{SIMULAÇÕES E RESULTADOS}

A Figura 4 apresenta a interface gráfica desenvolvida através de uma GUIDE do MATLab com o objetivo de facilitar a interação com o usuário. O software pode ser adquirido a partir do envio de $e$-mail para os autores.

Inicialmente, o usuário deverá inserir as condições iniciais da situação a ser simulada. A primeira janela deverá ser preenchida com as dimensões em $x$ e $y$ (juntamente com os seus incrementos $d x$ e $d y$, respectivamente) do objeto em questão.

A segunda janela é destinada para inserção de dados referentes ao material do objeto, são eles: condutividade térmica (k), massa específica (p) e calor específico (Cp).

Em seguida, no campo a ser preenchido deve constar o coeficiente convectivo (h) ao qual está submetido o material.

Partindo para o campo das constantes transientes, deverão ser inseridos o passo temporal (dt) e o tempo máximo de iterações que serão realizadas (tmáx).

Ainda sobre as condições iniciais, deverão ser digitadas as temperaturas laterais que estarão em contato com objeto na janela "Temperaturas", atentando-se para a particularidade do software, em que o objeto se encontra precisamente em contato com um fluido na sua lateral superior.
O botão "Permanente", ao ser pressionado, calcula e esboça a distribuição da temperatura em um gráfico $2 \mathrm{D}$, no tempo inicial $(\mathrm{t}=0)$.

O botão "Transiente", ao ser pressionado, apresenta a distribuição da temperatura no centro da barra com o decorrer do tempo, assim como o instante de tempo em que se encontra. A seleção do botão "Transiente" também gera uma representação 3D do gradiente de temperatura pela qual o objeto foi acometido. Ao final do número máximo de iterações, é exibido um gráfico $2 \mathrm{D}$, referenciando a variação de temperatura obtida, máxima e mínima. O botão "Parar", ao ser pressionado, permite a parada da simulação em execução.

$\mathrm{O}$ software desenvolvido possibilita ao usuário o acesso ao relatório de temperaturas alcançadas e o salvamento dos gráficos exibidos. O primeiro é apresentado na caixa "Relatório", e o segundo é permitido após a seleção do item "Figura 1", "Figura 2", "Figura 3" ou "Figura 4", na caixa "Figuras".

A Figura 4 apresenta valores para uma simulação inicial. Com o comprimento da barra de 0,2 e 0,3 metros, nas direções $x$ e $y$, respectivamente e supondo-se uma barra composta de ferro fundido, com condutividade térmica igual a $62,1(\mathrm{~W} / \mathrm{m} \cdot \mathrm{K})$, massa específica igual a $7870\left(\mathrm{~kg} / \mathrm{m}^{3}\right)$ e calor específico igual a $627(\mathrm{~J} / \mathrm{kg} \cdot \mathrm{K})$. O coeficiente convectivo é igual a $50\left(\mathrm{~W} / \mathrm{m}^{2} \cdot \mathrm{K}\right)$. O passo no 
tempo é 5 segundos, e o número de iterações máximas é 15000 segundos. As temperaturas nas laterais esquerda, direita e inferior é $500{ }^{\circ} \mathrm{C}$, e a superior está em contato com um fluido com $30 \mathrm{~K}$.

A Figura 5 apresenta o gráfico em 2D, referente ao regime permanente, ou seja, a distribuição de temperatura no tempo inicial $\mathrm{t}=$ 0 e, como era de se esperar, apresenta a temperatura das bordas de $500{ }^{\circ} \mathrm{C}$.

\section{Figura 5 - Distribuição de temperatura no regime} permanente

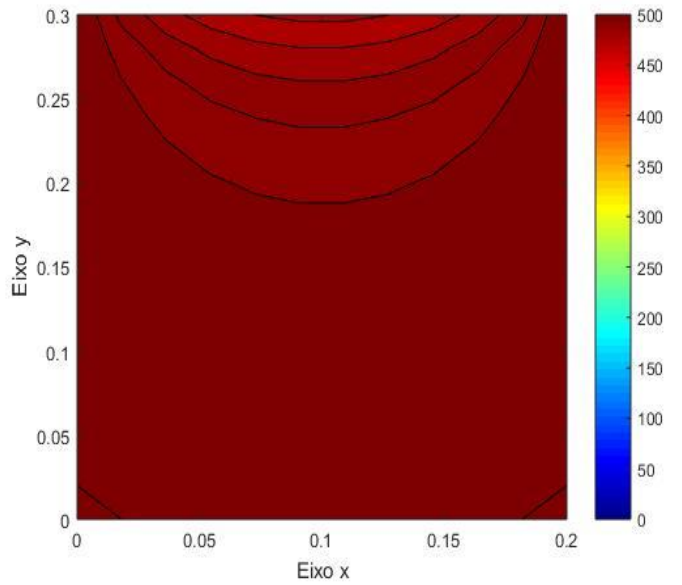

Fonte: acervo dos autores.

A Figura 6 ilustra o gráfico 2D obtido ao se pressionar o botão "Transiente", relativo à temperatura no centro da barra com o passar do tempo. Como não é possível apresentar o movimento nessa ocasião, apresenta-se a distribuição no instante $\mathrm{t}=15000$ segundos.

Figura 6 - Distribuição de temperatura no regime permanente

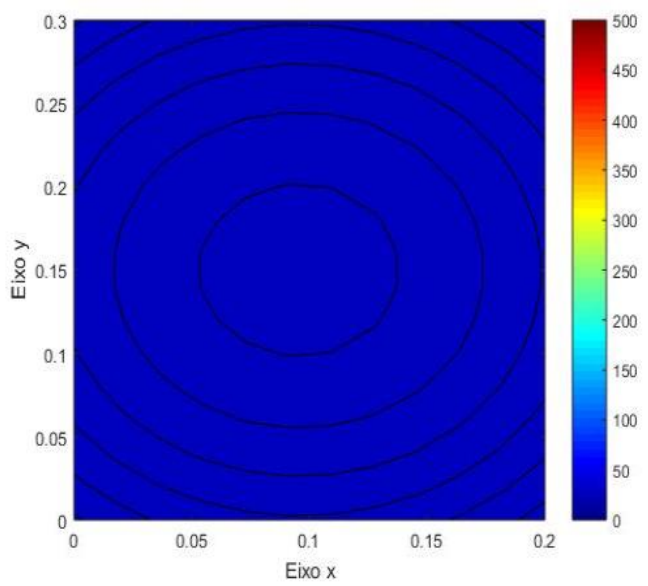

Fonte: acervo dos autores.
O gráfico 3D da posição em função da temperatura pode ser visto na Figura 7. Este gráfico exibe a variação da temperatura no decorrer do tempo em toda a barra e, assim como no gráfico anterior, é impossível de ser demonstrado aqui.

Figura 7 - Distribuição de temperatura no regime permanente

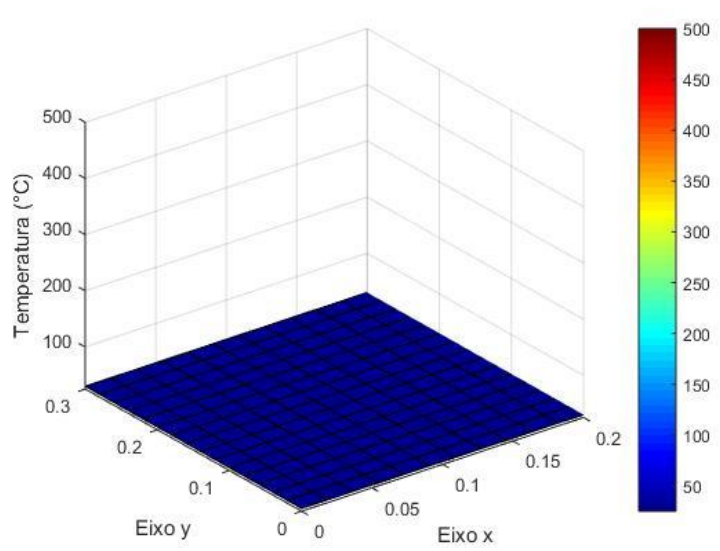

Fonte: acervo dos autores.

A Figura 8 é a representação $2 \mathrm{D}$ das temperaturas obtidas, que se inicia em $500{ }^{\circ} \mathrm{C}$ e, com o passar do tempo, com o resfriamento da barra, tende a se estabilizar por volta de $\mathrm{t}=$ 6000 segundos.

Figura 8 - Distribuição de temperatura no regime permanente

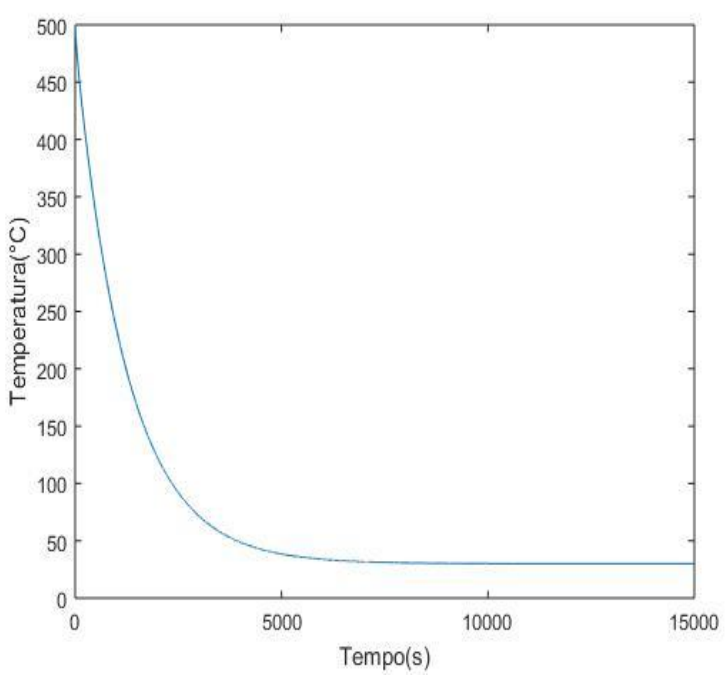

Fonte: acervo dos autores.

Ao final da simulação o software nos fornece as temperaturas máxima, mínima para os regimes permanente e transiente, e também 
de centro para este último, respectivamente. $\mathrm{O}$ relatório emitido para os dados utilizados é apresentado na Figura 9.

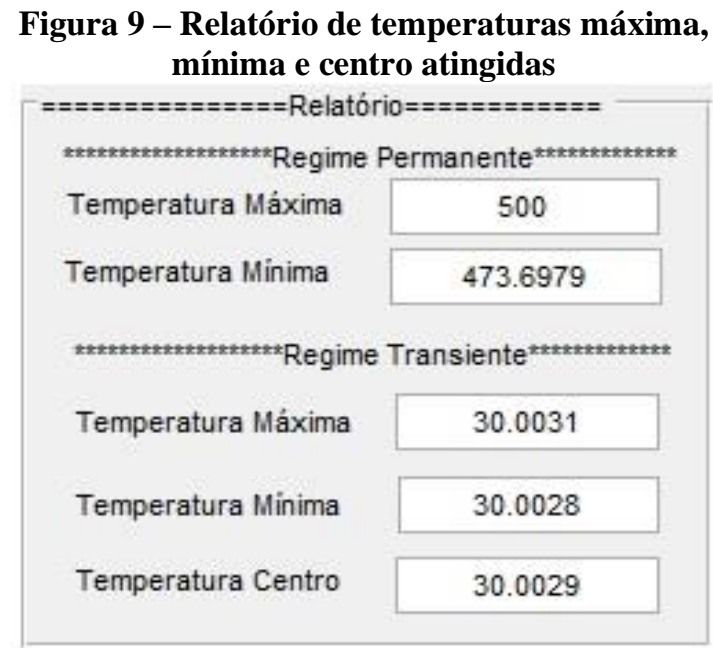

Fonte: acervo dos autores.

Por meio dos resultados obtidos pode-se validar o funcionamento da interface, visto que, de acordo com o esperado, as temperaturas atingidas no regime transiente chegam muito próximas à temperatura do fluido em contato com a barra. Ademais, no regime permanente a variação de temperatura acontece somente na região lateral superior onde está em contato com o fluido, visto que a temperatura do fluido é muito mais baixa que a temperatura dos nós adjacentes.

\section{CONCLUSÃO}

Neste trabalho objetivou-se o desenvolvimento de uma interface gráfica através do ambiente GUIDE do MATLab, para servir de material de apoio para estudantes de Engenharia em disciplinas nas quais o tema transferência de calor é abordado. O software possibilita a visualização de distribuição de temperaturas e o tempo de resfriamento, em função da geometria retangular e das propriedades do tipo de material. O software foi testado e os resultados apresentados neste trabalho foram coerentes com os encontrados na literatura. O software tem características open source, o que permite outros usuários fazerem melhorias em seu código fonte, acrescentando outras funções.

\section{REFERÊNCIAS}

ANSYS CFX. ANSYS CFX®. Disponível em: <https://www.ansys.com/>. Acesso em 03 mar. 2019.

INCROPERA, F. P.; DEWITT, D. P.; BERGMAN, T. L. Fundamentos de Transferência de Calor e Massa. Rio de Janeiro: LTC, 2008.

ÇENGEL, Y. A; GHAJAR, A. J. Transferência de Calor e Massa - Uma abordagem prática. Porto Alegre: AMGH, 2012.

LIMA, W. M. et al. Uma Ferramenta Computacional para Suporte nos Processos de Ensino e Aprendizagem de Equações Diferenciais Parciais. Revista de Ensino de Engenharia, [s.1.], v. 35, n. 1, p. 65-73, 2016.

MALISKA, C. R. TransCal V 1.1 (1998). Disponível em <http://www.sinmec.ufsc.br/site/softwares.ph p?id=2 > Acesso em: 04 mar. 2019.

PIERITZ, R. A. et al. CFD Sinflow library: a framework to develop engineering educational codes in CFD and thermal sciences. Comp. Applic. In Engineering Educator, v. 12, n. 1, p. 31-43, 2004.

SILVA, W. P; SILVA, C. D. P. S; LIMA, A. G. B. 1 D HeatTransfer: um software para simulações de problemas difusivosconvectivos unidimensionais transientes. Revista da Vinci, Curitiba, v. 3, n. 1, p. 171186, 2006.

Zin Cho, N. Particle Transport in Monte Carlo Method for Heat Conduction Problems. HeatConduction - Basic Research, 2011. 


\section{DADOS BIOGRÁFICOS DOS AUTORES}
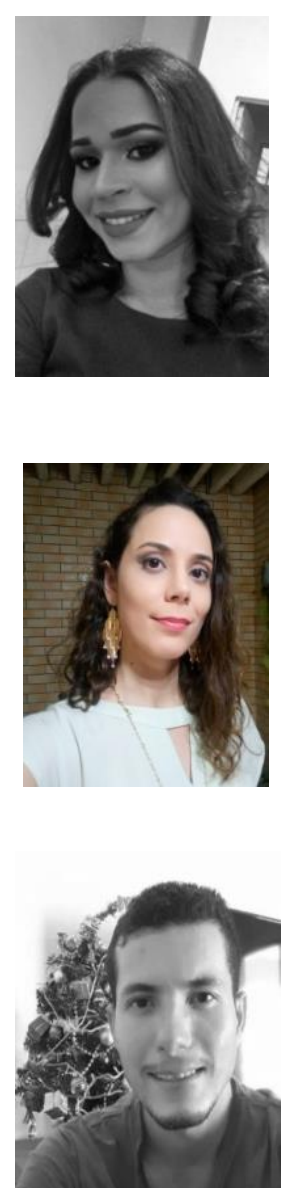

Marília Pereira Silva - Graduada em Bacharelado Interdisciplinar em Ciência e Tecnologia pela Universidade Federal do Maranhão (2017), graduada em Engenharia Civil pela Universidade Federal do Maranhão (2019).

Gislane Pinho de Oliveira - Professora Assistente da Universidade Federal do Maranhão, no BiCT- Engenharia Ambiental do Campus Balsas. Graduada em Engenharia Química da Universidade Federal do Rio Grande do Norte e Mestre em Engenharia Química pela Universidade Federal do Rio Grande do Norte com atuação em tratamento de efluentes industriais, adsorção, catálise ambiental, peneiras moleculares, fotodecomposição.

Raimundo Nonato Diniz Costa Filho - Possui graduação em Engenharia Elétrica pela Universidade Federal do Maranhão (UFMA) (2011). Mestrado (2013) e Doutorado (2018) em Engenharia Elétrica pela UFMA. Trabalhou de setembro de 2017 a janeiro de 2018 na Universidade de Dalhousie orientado pelo professor M. E. El-Hawary. Atualmente é Professor Adjunto da Universidade Federal do Maranhão- Campus Balsas. Suas atividades incluem o desenvolvimento de metodologias e software de análise para a operação e planejamento de Sistemas de Energia Elétrica (SEE), Controladores FACTS (Flexible AC Transmission Systems) e PSS (Power System Stabilizers), aplicações de técnicas de inteligência artificial no ajuste coordenado de controladores com vista a estabilidade angular em SEE e desenvolvimento de novas técnicas de inteligência artificial. É membro do IEEE (Institute of Electrical and Electronics Engineers). 